\title{
BMJ Open Protocol for a non-randomised pilot and feasibility study evaluating a multicomponent intervention to simplify medication regimens for people receiving community-based home care services
}

To cite: Sluggett JK, Page AT, Chen EYH, et al. Protocol for a non-randomised pilot and feasibility study evaluating a multicomponent intervention to simplify medication regimens for people receiving community-based home care services. BMJ Open 2019;9:e25345. doi:10.1136/ bmjopen-2018-025345

- Prepublication history and additional material for this paper are available online. To view these files, please visit the journal online (http://dx.doi. org/10.1136/bmjopen-2018025345).

Received 14 August 2018

Revised 5 June 2019

Accepted 7 June 2019

Check for updates

(c) Author(s) (or their employer(s)) 2019. Re-use permitted under CC BY-NC. No commercial re-use. See rights and permissions. Published by BMJ.

For numbered affiliations see end of article.

Correspondence to Dr Janet Kathleen Sluggett; janet.sluggett@monash.edu

\section{ABSTRACT}

Introduction Managing medication regimens is one of the most complex and burdensome tasks performed by older people, and can be prone to errors. People living with dementia may require medication administration assistance from formal and informal caregivers. Simplified medication regimens maintain the same therapeutic intent, but have less complex instructions and administration schedules. This protocol paper outlines a study to determine the feasibility of a multicomponent intervention to simplify medication regimens for people receiving community-based home care services.

Methods and analysis This is a non-randomised pilot and feasibility study. Research nurses will recruit 50 people receiving community-based home care services. All participants will receive the intervention from a clinical pharmacist, who will undertake medication reconciliation, assess each participant's capacity to self-manage their medication regimen and apply a structured tool to identify opportunities for medication simplification. The pharmacist will communicate recommendations regarding medication simplification to registered nurses at the community-based home care provider organisation. The primary outcome will be a description of study feasibility (recruitment and retention rates, protocol adherence and stakeholder acceptability). Secondary outcomes include the change in number of medication administration times per day, medication adherence, quality of life, participant satisfaction, medication incidents, falls and healthcare utilisation at 4 months.

Ethics and dissemination Ethical approval was obtained from the Monash University Human Research Ethics Committee and the community-based home care provider organisation's ethical review panel. Research findings will be disseminated to consumers and caregivers, health professionals, researchers and healthcare providers through the National Health and Medical Research Council Cognitive Decline Partnership Centre and through conference presentations, lay summaries and
Strengths and limitations of this study

- This study will provide new information regarding the burden of medication administration among older people receiving community-based home care services and inform future strategies to improve medication management.

- Quantitative and qualitative approaches will be used to comprehensively assess study outcomes.

- The outcomes to be assessed by this study are relevant to people receiving community-based home care services and their informal caregivers, community-based home care providers, clinicians and policy-makers.

- A potential limitation is that study participants will be recruited from one service provider which means findings may not be generalisable to all people receiving community-based home care services.

peer-reviewed publications. This study will enable an improved understanding of medication management and administration among people receiving community-based home care services. This study will inform the decision to proceed with a randomised controlled trial to assess the effect of this intervention.

Trial registration number ACTRN12618001130257; Preresults.

\section{INTRODUCTION}

Older people often have complex medication regimens to manage multiple chronic diseases. For example, two-thirds of Australians aged 75 years and older use five or more medications on a daily basis. ${ }^{1}$ The overall prevalence of medication use in the community setting is increasing, as evidenced 
by a doubling in the median number of medications prescribed to adults aged 65 years and over in the USA, from 2 to 4 medications, between 1988 and $2010 .^{2}$ The increasing prevalence of polypharmacy, together with a variety of factors such as increased availability of non-oral formulations and medication delivery devices, and special administration instructions for certain medications (eg, crush, take with food) means that medication management is one of the most complex medical tasks performed by community-dwelling older people and their formal and informal caregivers. ${ }^{3}$

Medication regimens with multiple administration times can be particularly burdensome for older people and/ or informal caregivers. ${ }^{3}$ Having a complex medication regimen is also associated with a range of adverse events among community-dwelling older people, including medication self-administration errors, ${ }^{4}$ unplanned hospitalisations ${ }^{5}$ and all-cause mortality. ${ }^{6}$ It is possible that undertaking medication regimen simplification could lead to a reduction in the number of times that medications need to be administered, and may reduce some of the burden associated with medication management in the community setting. Medication regimen simplification refers to the process of consolidating the number of administration times through strategies such as administering medications at the same time, using long acting in preference to short-acting formulations, and switching from multiple single ingredient to combination formulations, where possible. ${ }^{7}$

Evidence from previous studies suggests that people living with dementia and their informal caregivers often require practical support from health professionals to deal with medication-related issues. ${ }^{8}$ In accord, supporting people living with dementia and caregivers to simplify medication regimens for individuals living with dementia has been identified as an unmet priority. ${ }^{39}$ Simplifying medication regimens for community-dwelling people with dementia is particularly important because people living with dementia use at least as many, if not more, medications than people without dementia. ${ }^{10}{ }^{11}$ Because dementia can further impact the ability to plan, organise and undertake medication management, ${ }^{12}$ informal caregivers are often involved in medication management for people living with dementia. For example, in the US National Health and Aging Trends Study and National Study of Caregiving, informal caregivers who cared for a person living with dementia were $64 \%$ more likely to assist with ordering medications and $76 \%$ more likely to keep track of medications in comparison to caregivers for older people without dementia. ${ }^{13}$ Previous research suggests that people living with dementia and their informal caregivers are interested in interventions to reduce unnecessary challenges associated with medication use. ${ }^{14}$

An ongoing, multidisciplinary randomised controlled trial (RCT) is investigating an intervention to simplify medication regimens in South Australian residential aged care facilities. ${ }^{7}$ However, unlike in residential aged care settings, informal caregivers play a major role in determining medication administration schedules for community-dwelling people for whom they provide care. ${ }^{15}$ Furthermore, few people receiving community-based home care services have the direct support of a registered nurse to oversee all medication management and administration. Simplifying medication regimens is of interest to community-based home care service providers because multiple medication administration times may affect how much assistance older people require ${ }^{16}$ and because people who transition from care at home to residential aged care have increasingly complex medication regimens that are time consuming and costly to administer. ${ }^{17}$ However, the feasibility of simplifying medication regimens in this setting is currently unknown.

The aim of this pilot and feasibility study is to determine the feasibility of a multicomponent intervention to simplify medication regimens for people receiving community-based home care services. A quantitative and qualitative feasibility assessment will be undertaken to inform the decision to proceed with a RCT to assess the effect of this intervention.

The primary objectives of the pilot and feasibility study are as follows:

1. To determine how many people accept the invitation to participate in the study.

2. To determine how many participants receive the intervention.

3. To estimate recruitment and refusal rates, and 4-month follow-up rates.

4. To explore, qualitatively, the acceptability of the recruitment processes, assessments, intervention delivery and secondary outcome measures with key stakeholders.

5. To investigate the acceptability of the intervention to stakeholders by determining whether there are changes to medication regimens at 4 months after study entry.

The secondary objectives are:

1. To measure key domains for secondary outcome measures including percentage of participants who are able to self-report data for assessments, assessment completion rates by research nurses, missing data, estimates, variances and 95\% CIs for any comparisons.

2. To collect and synthesise secondary outcome data to inform the sample size estimation for a subsequent RCT, should this be feasible.

\section{METHODS AND ANALYSIS}

\section{Study design}

This pilot and feasibility study is a non-randomised study and all participants will receive the intervention. The pilot and feasibility study will be consistent with guidance proposed by Eldridge $e t a l^{18}$ and O'Cathain $e t a l,{ }^{19}$ and reported using the Standard Protocol Items: Recommendations for Interventional Trials reporting template (online supplementary file 1 ) ${ }^{20} \mathrm{~A}$ mixed methods approach will be undertaken to assess feasibility. 


\section{Governance and consultation}

The research team comprises nurses, pharmacists and a geriatrician with expertise in aged care research. The research team also comprises representatives from the community-based home care provider. The service provider is an active research partner, and informed the protocol design and implementation of the research.

The Project Governance Committee will comprise the chief investigators and nominated representatives from the organisation providing the community-based home care services. This committee will monitor compliance with the study protocol, data collection and data analysis throughout the study period. The Stakeholder Reference Group is a separate committee that will be comprised of health professionals, community-based home care services staff, people who receive aged care services and informal caregivers. The chief investigators and the community-based home care provider will determine membership of the Stakeholder Reference Group. The purpose of this group is to support the chief investigators to engage with a range of relevant stakeholders with different types of expertise and backgrounds related to the research study. The chief investigators will work closely with the community-based home care provider to conduct face-to-face consultations with other relevant stakeholders, including people who receive aged care services and informal caregivers, during the pre-intervention period.

\section{Patient and public involvement}

The research team conducted face-to-face consultations with the formal Consumer and Carer Reference Group maintained by the overarching aged care organisation to discuss the design, recruitment processes and conduct of the current pilot and feasibility study. This group comprises 10-12 people who either receive community-based home care services or residential aged care services from the aged care organisation, or have a family member who receive these services. This group was established in 2013 and meets regularly to provide advice on issues relating to services provided by the aged care organisation. The research team also consulted the consumer and carer members of the Stakeholder Reference Group of a related study. ${ }^{7}$ Feedback on the relevance of the outcome measures to be assessed in this study for older people living in the community was also sought during the face-to-face consultations.

\section{Context}

This pilot and feasibility study will involve people receiving a government-funded community-based home care service. These services include Commonwealth Home Support Programme services or Home Care Packages. Commonwealth Home Support Programme services provide entry-level ongoing support services and are targeted at older Australians who require assistance with one or more instrumental activities of daily living but can otherwise manage most aspects of their daily living. ${ }^{21}$ Individuals who apply for a Home Care Package undergo a structured assessment process, and are subsequently allocated to receive a funding package that ranges from basic care needs (level 1) through to high care needs (level 4). Both the Commonwealth Home Support Programme services and Home Care Packages are funded by the Australian Government and subsidise an approved community-based home care provider to provide services that meet each individual's care need. ${ }^{22}$ Services can include assistance with meals, transport, personal care and medication management. Development of community-based home care services is consistent with the Government's objective of providing services that facilitate older people to remain living independently in the community. ${ }^{17}$ Nearly one in five Australians receiving a government-subsidised community aged care service package are living with dementia. ${ }^{23}$

\section{Participants}

The participants will be 50 people receiving Commonwealth Home Support Programme services or Home Care Packages in metropolitan and rural South Australia. Individuals who are able to participate in structured assessments in English and are prescribed medication(s) to be administered two or more times daily on a regular basis are eligible. This means that a person prescribed one medication two times per day on a regular basis is potentially eligible to participate.

People estimated to have less than 3 months to live and those deemed by health professionals and caregivers to be medically unstable (eg, experiencing delirium) will be excluded. People may also be excluded for any other reason at the discretion of their regular treating clinicians or informal caregivers.

\section{Recruitment and consent process}

Research nurses were contracted by the community-based home care provider organisation and therefore had good knowledge of organisational processes, policies and procedures. A training package adapted from a related study conducted in residential aged care ${ }^{11}$ was used to train the research nurses in the consent process for the present study, and to train the research nurses and project pharmacist to use the data collection tools and administer assessments.

Participant recruitment commenced on 16 July 2018 and the first participant was enrolled on 22 August 2018. Research nurses liaised with community-based home care provider staff to obtain a list of all people receiving medication administration assistance from the organisation and who were potentially eligible. Research nurses then mailed information about the study to all people receiving medication administration assistance from the provider organisation. Research nurses then conducted follow-up telephone calls with all people who were mailed information to determine eligibility for the study, to provide verbal information about the study, and to seek written informed consent to participate. This process was performed in accordance with the ethical principles for 
involving people with cognitive impairment in research studies outlined in the Australian National Statement for Ethical Conduct in Human Research. ${ }^{24}$

For people who had the capacity to provide consent to participate, written consent was obtained directly from that individual. Online supplementary file 2 provides a model consent form. When the person was unable to provide written informed consent to participate, consent to participate was sought from the person's guardian, next of kin or significant other. For people with enduring power of guardianship, the research nurses explained as much about the study as possible to the person, and sought written informed consent from the guardian.

The Dementia Australia Guide to Dementia Friendly Language was used when writing about dementia or the impact of supporting a person living with dementia in study materials. ${ }^{25}$ Research nurses were trained to use the principles outlined in the Dementia Australia 'Talk to Me' tips for talking to people living with dementia during discussions with all participants, particularly when speaking with people living with dementia and when administering the Dementia Severity Rating Scale in third-party informant interviews. ${ }^{26}$ Using appropriate language that is accurate, respectful, inclusive, empowering and non-stigmatising is important when writing or talking about dementia. ${ }^{25}$

\section{Baseline data collection}

The research nurses will collect all data for each participant at baseline and 4 months after study entry as outlined in table 1 . The research nurses will speak with the participant and/or their informal caregivers, and liaise with the community pharmacy, general medical practitioner (GP) and the community-based home care provider to obtain information about prescribed, dispensed and non-prescription medications. Demographic and clinical data will be sourced from the community-based home care provider nursing progress notes. The research nurses will contact the participant's GP to source information from the participant's medical record, including current and past health conditions, adverse drug reactions, demographic and social information, any hospital discharge summaries and details of any Home Medicines Reviews (HMRs) performed in the 4 months prior to study entry. Other data will be collected via participant self-report and the research nurses conducting interviews with thirdparty informants (table 1). Participants who are unable to complete assessments outlined in table 1 are still eligible for inclusion in the study. Third-party informants will be staff members or informal caregivers who have known the participant for at least 2 weeks. Research nurses will be trained to ask informants to advise the research nurses if they feel they do not know the participant well enough to answer any specific questions.

\section{Intervention}

The intervention will be delivered after recruitment and baseline data collection (table 2) by a project pharmacist who is accredited to perform HMRs. The three main components of the intervention are outlined in table 2.

\section{Medication reconciliation}

The first step in the intervention will involve the project pharmacist undertaking medication reconciliation. Medication reconciliation is the process of developing the "best possible medication history' that outlines which medications the participant actually takes, the dose form, the dose of each medication and the corresponding administration schedule. ${ }^{27} 28$ This step is necessary because discrepancies often exist between medication records maintained by aged care providers, GPs, pharmacies and participants. ${ }^{29}$ For example, medications prescribed by medical practitioners other than the regular GP, complementary and alternative medications and overthe-counter medications may be omitted from GP medication records. Conversely, discontinued medications may continue to be included in GP medication records. A preliminary medication history will be compiled by the project pharmacist prior to visiting each participant. The preliminary medication history will be based on information collected from the participant's community pharmacy, GP and community-based home care provider by the research nurse. This information will be verified by the project pharmacist during a participant interview to ensure that any medications independently managed by the participant and not included on the community pharmacy, GP or community-based home care provider list are included in the final version of the best possible medication history prepared by the project pharmacist. This step will ensure that the subsequent recommendations to simplify the medication regimen are based on a full and accurate understanding of the participant's current medication regimen. The importance of having an accurate medication history available prior to medication simplification is outlined in the Medication Regimen Simplification Guide for Residential Aged CarE (MRS GRACE). ${ }^{30}$

\section{Capacity to self-manage}

The second step in the intervention will involve the project pharmacist assessing the participant's ability to self-manage their own medication regimen by applying the Drug Regimen Unassisted Grading Scale (DRUGS). ${ }^{31}$ This is because it is important to understand a person's capacity to self-manage their medication regimen before recommending changes to their medication administration routine. The DRUGS criteria have four assessed domains for each medication taken that considers identification, access, dosage and timing. ${ }^{31}$ It has demonstrated inter-rater reliability and test-retest reliability when used among people aged 70 years and older residing in retirement villages. ${ }^{31}$ The DRUGS criteria require the participant to be able to correctly identify the medication, dose and administration times. The participant is then asked to demonstrate the process of accessing the dose by opening the medication container, obtaining the dose from a dose administration aid (eg, blister pack, dosette 
Table 1 Overview of data collection at baseline and 4 months post study entry

\begin{tabular}{|c|c|c|c|c|}
\hline \multirow[b]{2}{*}{ Item } & \multirow[b]{2}{*}{$\begin{array}{l}\text { Description or tool used to measure the } \\
\text { outcome }\end{array}$} & \multirow[b]{2}{*}{ Data source } & \multicolumn{2}{|c|}{ Data collection time points } \\
\hline & & & Baseline & $\begin{array}{l}4 \text { months post } \\
\text { study entry }\end{array}$ \\
\hline Demographics & Age, sex, living arrangements & Community-based home care provider & $\checkmark$ & \\
\hline Aged care package & $\begin{array}{l}\text { The commencement date, the service type } \\
\text { and package level will be identified. }\end{array}$ & - Community-based home care provider & $\checkmark$ & $\checkmark$ \\
\hline $\begin{array}{l}\text { No of home visits in } \\
\text { the preceding week }\end{array}$ & $\begin{array}{l}\text { Number of community-based home care } \\
\text { provider visits over the last } 7 \text { days will be } \\
\text { extracted. }\end{array}$ & Community-based home care provider & $\checkmark$ & $\checkmark$ \\
\hline Medical conditions & $\begin{array}{l}\text { Diagnoses of medical conditions, } \\
\text { syndromes and diseases will be } \\
\text { determined from multiple sources. }\end{array}$ & $\begin{array}{l}\text { Participant and third-party informant } \\
\text { General medical practitioner } \\
\text { Community-based home care provider }\end{array}$ & $\checkmark$ & $\checkmark$ \\
\hline Medications taken & $\begin{array}{l}\text { Data relating to prescribed medications, } \\
\text { medication use and previous adverse } \\
\text { drug events will be reconciled from } \\
\text { multiple sources. Details of all prescription } \\
\text { and non-prescription medications, } \\
\text { including complementary and alternative } \\
\text { medications, administered regularly and as } \\
\text { required will be recorded. The medication } \\
\text { name, strength, number of dosing times } \\
\text { and administration details will be recorded. }\end{array}$ & $\begin{array}{l}\text { Participant and third-party informant } \\
\text { General medical practitioner and } \\
\text { community pharmacist } \\
\text { Community-based home care provider }\end{array}$ & $\checkmark$ & $\checkmark$ \\
\hline $\begin{array}{l}\text { Home Medicines } \\
\text { Review (HMR) }\end{array}$ & $\begin{array}{l}\text { An HMR is an Australian Government } \\
\text { remunerated service undertaken } \\
\text { collaboratively by pharmacists accredited } \\
\text { to conduct HMRs and GPs, to identify and } \\
\text { resolve medication-related problems for } \\
\text { people living in the community. }{ }^{40} \text { Dates } \\
\text { that any HMRs were provided will be } \\
\text { determined using multiple sources. People } \\
\text { who have previously received an HMR } \\
\text { remain eligible for inclusion in this study. }\end{array}$ & $\begin{array}{l}\text { Participant and third-party informant } \\
\text { General medical practitioner and } \\
\text { community pharmacist } \\
\text { Community-based home care provider }\end{array}$ & $\boldsymbol{J}^{*}$ & $\checkmark$ \\
\hline $\begin{array}{l}\text { Adherence to } \\
\text { medication regimen }\end{array}$ & $\begin{array}{l}\text { Adherence to the medication regimen } \\
\text { will be assessed using the } 13 \text {-item Self- } \\
\text { Efficacy for Appropriate Medication use } \\
\text { Scale (SEAMS). }{ }^{33} \text { The SEAMS has been } \\
\text { demonstrated to be valid and reliable } \\
\text { among people with a variety of chronic } \\
\text { health conditions and among people with } \\
\text { low literacy. }{ }^{33} \text { There is a strong correlation } \\
\text { between measurement of self-efficacy with } \\
\text { the SEAMS and medication adherence as } \\
\text { assessed by the Morisky Scale. }{ }^{33}\end{array}$ & $\begin{array}{l}\text { Participant or third-party informant } \\
\text { interview }\end{array}$ & $\checkmark$ & $\checkmark$ \\
\hline Frailty & $\begin{array}{l}\text { Frailty status will be assessed using the } \\
5 \text {-item FRAIL screening test scale that } \\
\text { assesses fatigue, resistance, ambulation, } \\
\text { illnesses and recent weight loss. }{ }^{41} \text { The } \\
\text { FRAIL is relatively short and can be easily } \\
\text { administered in clinical and research } \\
\text { settings to screen for frailty. Possible } \\
\text { scores range from } 0 \text { to } 5 \text {, with a score of } 0 \\
\text { indicating robust health status, scores of } \\
1-2 \text { indicating the person is prefrail and a } \\
\text { score of } 3 \text { or more indicating the person } \\
\text { is frail. }{ }^{41} \text { A recent meta-analysis of three } \\
\text { studies showed prefrailty and frailty, as } \\
\text { defined by the FRAlL Scale, are associated } \\
\text { with increased mortality. }{ }^{42}\end{array}$ & $\begin{array}{l}\text { Participant or third-party informant } \\
\text { interview }\end{array}$ & $\checkmark$ & $\checkmark$ \\
\hline
\end{tabular}


Table 1 Continued

\begin{tabular}{|c|c|c|c|c|}
\hline \multirow[b]{2}{*}{ Item } & \multirow[b]{2}{*}{$\begin{array}{l}\text { Description or tool used to measure the } \\
\text { outcome }\end{array}$} & \multirow[b]{2}{*}{ Data source } & \multicolumn{2}{|c|}{ Data collection time points } \\
\hline & & & Baseline & $\begin{array}{l}4 \text { months post } \\
\text { study entry }\end{array}$ \\
\hline Dementia severity & $\begin{array}{l}\text { Assessed using the } 12 \text {-item Dementia } \\
\text { Severity Rating Scale (DSRS), }{ }^{43} \text { which } \\
\text { provides a validated measure of } \\
\text { impairment across the major functional } \\
\text { and cognitive domains. The DSRS } \\
\text { is recommended by the Australian } \\
\text { Government Dementia Outcomes } \\
\text { Measurement Suite for assessing the } \\
\text { severity and progression of dementia. }{ }^{44} \text { The } \\
\text { DSRS has excellent ability to distinguish } \\
\text { between people without cognitive } \\
\text { impairment and people with mild cognitive } \\
\text { impairment or Alzheimer's disease. }{ }^{44} \text { The } \\
\text { DSRS is written in a multiple choice format } \\
\text { and it can be administered in } 4-5 \text { min. }{ }^{44}\end{array}$ & Third-party informant interview & $\checkmark$ & $\checkmark$ \\
\hline Quality of life & $\begin{array}{l}\text { Quality of life will be assessed using the } \\
\text { Quality of Life in Alzheimer's Disease } \\
\text { (QoL-AD) Scale, }{ }^{34} \text { which is recommended } \\
\text { in the Australian Government Dementia } \\
\text { Outcomes Measurement Suite. }{ }^{44} \text { Each } \\
\text { of the } 13 \text { measures is rated on a scale of } \\
\text { one to four, with lower scores indicating } \\
\text { reduced quality of life. The QoL-AD Scale } \\
\text { was found to be reliable and valid when } \\
\text { administered to people living with dementia } \\
\text { who have MMSE scores greater than } 10 \\
\text { in a previous study in which } 155 \text { people } \\
\text { completed the QoL-AD Scale. }\end{array}$ & $\begin{array}{l}\text { Participant or third-party informant } \\
\text { interview }\end{array}$ & $\checkmark$ & $\checkmark$ \\
\hline Incidents & $\begin{array}{l}\text { Incidents that are routinely collected by } \\
\text { the community-based home care provider } \\
\text { will be captured. Falls will be defined as } \\
\text { 'events that results in a person coming } \\
\text { to rest inadvertently on the ground or } \\
\text { floor or other lower level'. }{ }^{47} \text { Details of } \\
\text { all falls, including the date of the fall, } \\
\text { severity and related health outcomes (eg, } \\
\text { fracture, hospitalisation) will be recorded. } \\
\text { Medication incidents will be recorded, } \\
\text { noting: the date of the medication incident, } \\
\text { the type of incident (eg, prescribing error, } \\
\text { pharmacy dispensing error identified by } \\
\text { staff, participant error, administration error } \\
\text { or adverse drug reaction) and severity. }\end{array}$ & $\begin{array}{l}\text { Community-based home care provider } \\
\text { Participant or third-party informant } \\
\text { interview }\end{array}$ & $\mathcal{J}^{*}$ & $\checkmark$ \\
\hline $\begin{array}{l}\text { Ambulance call- } \\
\text { outs }\end{array}$ & $\begin{array}{l}\text { Ambulance call-outs with and without } \\
\text { transportation will be determined. The date } \\
\text { and the reason(s) will be recorded. }\end{array}$ & $\begin{array}{l}\text { General medical practitioner and South } \\
\text { Australian Ambulance Service records } \\
\text { Community-based home care provider } \\
\text { Participant or third-party informant } \\
\text { interview }\end{array}$ & $J^{*}$ & $\checkmark$ \\
\hline
\end{tabular}


Table 1 Continued

\begin{tabular}{|c|c|c|c|c|}
\hline \multirow[b]{2}{*}{ Item } & \multirow[b]{2}{*}{$\begin{array}{l}\text { Description or tool used to measure the } \\
\text { outcome }\end{array}$} & \multirow[b]{2}{*}{ Data source } & \\
\hline & & & Baseline & $\begin{array}{l}4 \text { months post } \\
\text { study entry }\end{array}$ \\
\hline Hospital visits & $\begin{array}{l}\text { Emergency department visits that do not } \\
\text { result in admission and hospital admissions } \\
\text { will be determined. The date and the } \\
\text { reason(s) will be recorded. }\end{array}$ & $\begin{array}{l}\text { General medical practitioner and South } \\
\text { Australian Ambulance Service records } \\
\text { Community-based home care provider } \\
\text { Participant or third-party informant } \\
\text { interview }\end{array}$ & $\mathscr{V}^{*}$ & $\checkmark$ \\
\hline $\begin{array}{l}\text { Residential aged } \\
\text { care facility } \\
\text { admission }\end{array}$ & $\begin{array}{l}\text { Admission to a residential aged care } \\
\text { facility will be defined as either respite or } \\
\text { permanent accommodation. }\end{array}$ & $\begin{array}{l}\text { Community-based home care provider } \\
\text { Participant or third-party informant } \\
\text { interview }\end{array}$ & $\checkmark \dagger$ & $\checkmark$ \\
\hline All-cause mortality & $\begin{array}{l}\text { Any deaths in the } 4 \text { months after study } \\
\text { entry will be determined, and the date of } \\
\text { death recorded. }\end{array}$ & $\begin{array}{l}\text { Community-based home care provider } \\
\text { Third-party informant interview }\end{array}$ & & $\checkmark$ \\
\hline
\end{tabular}

${ }^{*}$ Data relating to these variables will be extracted for events in the 4 months prior to study entry.

†Data relating to admissions for respite care in the 4 months prior to study entry will be extracted.

FRAIL, Fatigue, Resistance, Ambulation, Illnesses, \& Loss of Weight; MMSE, Mini-Mental State Exam.

box), or correctly using the device (eg, insulin injection or metered-dose inhaler). This process is repeated for each medication in the participant's medication regimen. The DRUGS criteria will be scored by the project pharmacist in accordance with the published literature ${ }^{31}$ and the resulting score will be expressed as a percentage to provide a numerical score on a scale of $0-100$. If after applying the DRUGS criteria the project pharmacist believes the participant does not have sufficient capacity to self-manage the participant's medication regimen,

Table 2 Schedule of enrolment, intervention and assessments for the study

\begin{tabular}{|c|c|c|c|c|}
\hline \multirow{3}{*}{ Timepoint } & \multicolumn{4}{|c|}{ Study period } \\
\hline & \multirow{2}{*}{$\begin{array}{l}\text { Enrolment } \\
-t_{1}\end{array}$} & \multicolumn{2}{|l|}{ Post enrolment } & \multirow{2}{*}{$\begin{array}{l}\text { Close-out } \\
4 \text { months }\end{array}$} \\
\hline & & Baseline & Intervention & \\
\hline \multicolumn{5}{|l|}{ Enrolment } \\
\hline Eligibility screen & $x$ & & & \\
\hline Informed consent & $\mathrm{x}$ & & & \\
\hline \multicolumn{5}{|l|}{ Assessments } \\
\hline Demographic information & & $\mathrm{X}$ & & \\
\hline Aged care package details and services accessed & & $x$ & & $x$ \\
\hline Medical conditions & & $\mathrm{x}$ & & $\mathrm{x}$ \\
\hline Medication use & & $x$ & & $\mathrm{x}$ \\
\hline Self-Efficacy for Appropriate Medication use Scale & & $x$ & & $\mathrm{x}$ \\
\hline FRAIL screening test & & $x$ & & $x$ \\
\hline Dementia Severity Rating Scale & & $\mathrm{X}$ & & $\mathrm{x}$ \\
\hline Katz Activities of Daily Living & & $x$ & & $\mathrm{x}$ \\
\hline Quality of Life in Alzheimer's Disease & & $x$ & & $\mathrm{x}$ \\
\hline Short Assessment of Patient Satisfaction & & $x$ & & $x$ \\
\hline Incidents & & $x$ & & $\mathrm{x}$ \\
\hline Ambulance call-outs and hospital visits & & $x$ & & $\mathrm{x}$ \\
\hline Residential aged care facility admissions & & $x$ & & $\mathrm{x}$ \\
\hline Date of death & & & & $x$ \\
\hline \multicolumn{5}{|l|}{ Interventions } \\
\hline Medication reconciliation & & & $x$ & \\
\hline Capacity to self-manage medications & & & $\mathrm{x}$ & \\
\hline $\begin{array}{l}\text { Medication regimen simplification assessment and } \\
\text { communication of recommendations }\end{array}$ & & & $\mathrm{x}$ & \\
\hline
\end{tabular}


this will be brought to the attention of a registered nurse employed by the community-based home care provider. After discussing with the registered nurse, the project pharmacist may contact the participant's regular GP and pharmacist to discuss management strategies if the participant is self-managing part or all of their medication regimen and the pharmacist believes the participant does not have sufficient capacity to do so.

\section{Medication regimen simplification}

The third step in the intervention will involve the application of a five-step, implicit structured tool to support decisions to simplify medication regimens. The pharmacist will use a modified version of MRS GRACE that was originally designed, validated and tested in the residential aged care setting. ${ }^{730}$ In order to modify the tool for application in the community setting, a multidisciplinary stakeholder meeting was convened in December 2017. Stakeholders included members of the expert panel who informed the development of MRS GRACE, and members of the research governance committee and stakeholder reference committee for the pilot and feasibility study. Minor revisions to the wording of the MRS GRACE tool and associated explanatory statement to support application in the community setting were made based on consultations and feedback from these stakeholders.

The project pharmacist's recommendations to simplify each participant's medication regimen will be informed by the medication reconciliation and assessment of participant's capacity to self-manage medications, medication adherence and the participant's clinical and functional profile. The participant's willingness to have their medication regimen simplified will be assessed during the pharmacist interview. The project pharmacist will prepare a written report for the community-based home care provider that includes the reconciled best possible medication history and a 'medication management statement' comprising assessments of adherence, capacity to selfmanage and recommendations for simplification. The project pharmacist will then discuss the opportunity for regimen simplification with a registered nurse employed by the community-based home care provider. After discussing with the registered nurse, the project pharmacist may contact the participant's regular GP and pharmacist where necessary to discuss strategies for simplifying the medication regimen. Strategies recommended by the pharmacist may include a change in medication administration time, formulation (eg, switching to a combination product or slow release preparation) or active ingredient. Recommended changes in medication administration times will be approved by the registered nurse employed by the community-based home care service providing care to the participant or the participant's GP prior to implementation. The GP will have the opportunity to review all other recommended simplification strategies before implementation and adjust the medication regimen accordingly. The participant's GP and/or community-based home care services nursing staff will ensure the participant or their guardian, next of kin or significant other are involved in the decision-making process before simplification strategies are implemented. At the end of the 'simplification' interview, the project pharmacist may provide the participant with a copy of their current medication list where clinically indicated or requested, and if no further recommendations for simplification require consideration by the community-based home care services nursing staff or the GP. If the project pharmacist identifies a clinically significant medication-related problem that poses risk of harm to the participant, this will be brought to the attention of the home care service provider and the participant's regular GP. If the project pharmacist believes that the participant would benefit from an HMR, this recommendation will be communicated in writing to the registered nurse employed by the community-based home care provider, who will forward this recommendation to the participant's regular GP.

\section{Concomitant care}

Participants will remain eligible for all usual care services during the study period. These services include, but are not limited to, clinical and care services provided by the community-based home care provider, community pharmacist, GP, medical specialists and other health service utilisation (eg, HMRs).

\section{Outcome measures}

Primary outcome

The primary outcome will be a narrative description of study feasibility, including recruitment and retention, protocol adherence and stakeholder acceptability. The primary outcome will be assessed using a mixed methods approach.

A document analysis will be undertaken to determine the number of people who were mailed information about the study, the number of people approached by telephone to participate in the study, the number of people who met the inclusion criteria and the number(s) of people who provided informed consent, received the intervention and completed 4-month follow-up. Quantitative data will be collected from field notes maintained by the project team, recruitment logs, the medication simplification intervention reports written by the project pharmacist, meeting minutes and email correspondence.

A qualitative approach will be used to determine (1) protocol adherence and adaptations, (2) acceptability of the recruitment processes, assessments, intervention delivery and secondary outcome measures and (3) barriers and enablers to intervention delivery. Facilitators and barriers important for the design of a subsequent RCT will also be explored. Qualitative data will be generated from small face-to-face focus groups or semistructured interviews undertaken after the intervention with approximately 10-15 key stakeholders in total. People participating in the study, family members who provided third-party consent for a person to participate, the research nurses, the project pharmacist, staff employed 
by the community-based home base provider organisation and GPs will be invited to participate. At least three study participants will be interviewed, including at least one person for whom simplification was not possible, one person for whom medication simplification was recommended but not implemented, and a person for whom at least one recommendation was implemented. The interview guide will comprise questions modified from that used in previous related research. ${ }^{7}$ All focus groups and semistructured interviews will be conducted face to face by two members of the research team. The sessions will be audiotaped, transcribed and analysed thematically using the procedures outlined by Braun and Clarke. ${ }^{32}$

\section{Secondary outcomes}

Secondary outcomes include the change in the number of medication administration times over a 24 hours period for regular medications at 4 months post study entry. This outcome was selected because (1) the main focus of medication regimen simplification is to reduce the number of dosing times and (2) the majority of medication simplification recommendations made by pharmacists in other studies ${ }^{1130}$ would lead to a reduction in the number of dosing times when implemented.

Medication administration times are defined as each separate occasion that medication is administered. It is anticipated that dose times may be expressed differently across different data sources used to develop the best possible medication history. For example, dose times may be reported using a 24 hours clock (eg, 06:00, 12:00, 18:00, 22:00), time slots (eg, 07:00-10:00, 12:00-14:00) or words (eg, breakfast, lunch, dinner, bedtime). These times will be standardised in our dataset and considered by the project pharmacist when ascertaining medication administration times. The number of distinct medication administration times will be counted and reported numerically. A hypothetical example of a medication regimen at baseline and 4-month follow-up that illustrates a change in the number of medication administration times is depicted in table 3 .

Other secondary outcomes at 4-month follow-up will be the total number of individual medication doses administered over a 24 hour period for regular medications; adherence to the medication regimen measured with the Self-Efficacy for Appropriate Medication use Scale ${ }^{33}$; quality of life using the Quality of Life in Alzheimer's Disease $\mathrm{Scale}^{34}$; participant satisfaction using the Short Assessment of Patient Satisfaction ${ }^{35}$; falls and medication incidents, as outlined in table 1.

The healthcare utilisation outcomes will be ambulance call-outs, emergency department visits, hospital admissions, admissions to residential aged care facilities and all-cause mortality (table 1 ). We will also consider the changes recommended by the project pharmacist, pharmacist time and time spent administering medications.

- Recommended changes to simplify the regimen will be extracted from the project pharmacist's deidentified report to the GP. These changes will consider the dose forms and medications that are most commonly recommended to be changed to simplify the regimen.

- Pharmacist time measured using the project pharmacist's record of the amount of time spent reconciling medications, identifying opportunities for simplification, generating recommendations and communicating with key stakeholders (eg, the participant, the participant's family, caregivers, GPs and community pharmacists). This information will be recorded prospectively as the project pharmacist delivers the intervention.

- Time spent administering medications will be estimated. This estimate will be informed by current medication

Table 3 Hypothetical medication regimen illustrating a reduction in the number of medication administration times for regular medications at follow-up

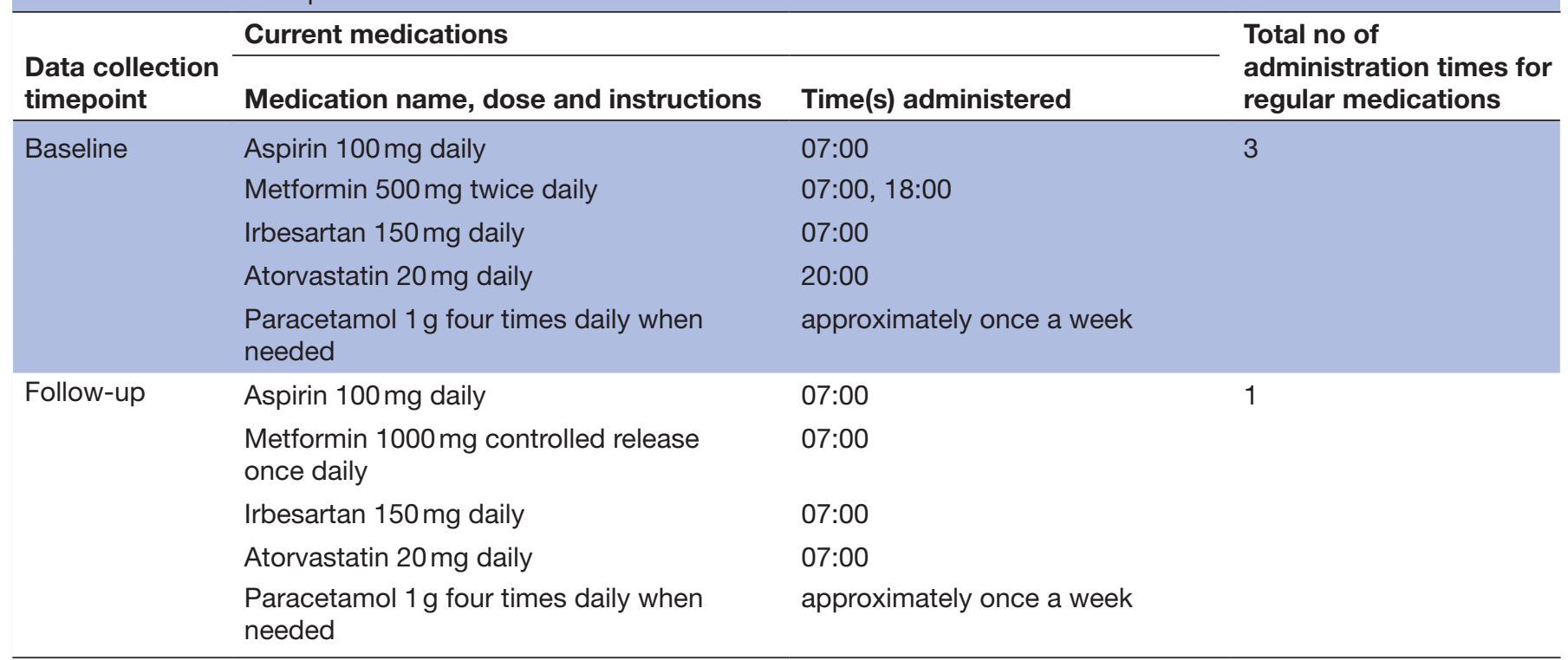


administration practices by the community-based home care provider. This estimate will account for various factors including the number of medications administered, administration times, dosage formulations and dose administration aids.

Where possible, we will compare the baseline medication history held by the community-based home care provider with the baseline best possible medication history independently prepared by the project pharmacist and identify, document and classify the clinical significance of any discrepancies. Any suspected and actual adverse events will be reported and analysed. These outcomes will inform the data collection methods for the planned subsequent RCT.

\section{Sample size}

As this is a pilot and feasibility study, a formal sample size calculation is not required. We will aim to recruit a sample size of 50 participants to assess feasibility across a diverse range of participants including those with and without a dementia diagnosis, different care needs or frailty status and with or without informal carers. We estimate that this target sample size would be greater than $10 \%$ of the number of people required for a future RCT. This target sample size is in keeping with the median target sample size of 30 (IQR 20-45) people for pilot studies, 36 (IQR 25-50) people for feasibility studies and 49 (IQR 36-61) people for pilot and feasibility studies observed in an audit of 79 pilot and feasibility RCTs registered on the United Kingdom Clinical Research Network database in $2012 .^{36}$

\section{Statistical analysis}

Quantitative data will be imported into the SAS Statistical Software (SAS Institute) for analysis and reported using descriptive statistics. Changes in the secondary outcome measures at follow-up will be assessed using the paired t-test (continuous variables) and McNemar's test (categorical variables).

\section{Data management}

Study participants will be assigned a unique code to enable data linkage throughout follow-up. All data will be entered into an electronic management system by research team members who are trained in data entry. Medications will be classified using WHO Anatomical and Therapeutic Chemical classification. To ensure accurate data entry, a second member of the research team will check data entry for the first 10 participants, and for a random $10 \%$ sample of records entered after that. Data collection and study conduct will be monitored through weekly meetings with the research team, representatives from the community-based home care provider organisation, research nurses and project pharmacist to ensure protocols are implemented consistently. Data collected as part of this study will be treated confidentially and stored securely at the Centre for Medicine Use and Safety, Faculty of Pharmacy and Pharmaceutical Sciences,
Monash University. Only study investigators based at the Centre for Medicine Use and Safety, Faculty of Pharmacy and Pharmaceutical Sciences, Monash University will have access to the final dataset.

\section{DISCUSSION}

This study will improve understanding of medication administration among older people receiving community-based home care services and inform strategies to improve medication management in this setting. Extensive stakeholder consultation has informed the study design and protocol development. An existing validated tool for use in the residential aged care setting was modified for use in the community setting, and it will be applied in this community-based study.

The intervention in this study comprises medication reconciliation, assessment of the ability of an individual to self-manage their own medication regimen, and identifying opportunities to simplify the medication regimen. This study will seek to establish the probable 'active components' of the intervention ${ }^{37}$ and to determine appropriate and acceptable outcome measures so that these parameters can be measured during a subsequent RCT. As such, all people included in this pilot and feasibility study will receive the intervention. A limitation with this approach is that the randomisation method for application in a subsequent RCT cannot be tested in the present study.

The intervention will be delivered by a single project pharmacist with considerable expertise in medication reconciliation. However, there are tools available to support a standardised approach to medication history taking $^{38}$ and a more structured approach will be considered in the design of a future RCT. The intervention will provide important information on the amount of time spent by the project pharmacist to deliver the intervention, but a limitation is that we will not be able to capture the time spent by the community-based home care provider staff, GPs and community pharmacists who will be involved in the implementation of the pharmacist's recommendations.

Study participants will be recruited from a cohort receiving community-based home care services from one service provider, so the study findings may not be generalisable, particularly to older people living independently without caregiver support. Asking people to self-report medication self-efficacy and adherence is another potential study limitation. However, previous studies of people with and without dementia in aged care settings have demonstrated a considerable proportion of people living with dementia are able to self-report outcomes ${ }^{39}$ and it is important to determine on an individual basis whether an individual is able to self-report outcomes. In the present study, a third-party informant may be interviewed when a participant is not able to undertake assessments themselves. Research nurses will also liaise with the participant's community pharmacist to provide a recent 
dispensing history and this will aid insight into adherence to prescription medications.

Complex medication regimens are often challenging for older people to manage, and considerable support with medication management is often needed. ${ }^{3}$ Complex regimens are associated with medication errors and poor adherence, as well as poor health outcomes. ${ }^{4-6}$ This study will determine the feasibility of a multicomponent intervention to simplify medication regimens for people receiving community-based home care services, which will inform the development and implementation of a subsequent RCT, should this be feasible.

\section{Author affiliations}

${ }^{1}$ Centre for Medicine Use and Safety, Monash Institute of Pharmaceutical Sciences, Monash University, Parkville, Victoria, Australia

${ }^{2}$ National Health and Medical Research Council (NHMRC) Cognitive Decline Partnership Centre, Hornsby Ku-ring-gai Hospital, Hornsby, New South Wales, Australia

${ }^{3}$ Department of Epidemiology and Preventive Medicine, School of Public Health and Preventive Medicine, Monash University, Melbourne, Victoria, Australia

${ }^{4}$ Research and Development, Helping Hand Aged Care, North Adelaide, South Australia, Australia

${ }^{5}$ School of Pharmacy and Medical Sciences, University of South Australia, Adelaide, South Australia, Australia

${ }^{6}$ Kolling Institute of Medical Research, Royal North Shore Hospital, Northern Clinical School, Faculty of Medicine and Health, The University of Sydney, Sydney, New South Wales, Australia

Contributors JKS, SH and JSB conceived the study and obtained grant funding. JKS, ATP and JSB participated in the design of the study protocol; they drafted the protocol and wrote the protocol manuscript. All authors critically reviewed the study protocol. MC, JVE, MH, TC, MA and CEO assisted in the development and implementation of the study. The final trial datasets will be collated and analysed by JKS, CEO, JI and EYHC. All authors critically reviewed and approved the final manuscript.

Funding This work is supported by the NHMRC Partnership Centre: Dealing with Cognitive and Related Functional Decline in Older People (known as the Cognitive Decline Partnership Centre (CDPC)) (grant number GNT9100000). The CDPC receives support from the NHMRC and funding partners including Helping Hand Aged Care, Hammond Care, Brightwater Care Group and Dementia Australia. The contents of the published materials are solely the responsibility of the individual authors identified and do not reflect the views of the NHMRC and any other funding bodies or the funding partners.

Competing interests This study was funded through the NHMRC CDPC and the community-based home care provider involved in this research is one of the funding partners of the NHMRC CDPC. JKS and JI are supported by NHMRC Early Career Fellowships. EYHC is supported by a postgraduate research scholarship funded through the NHMRC CDPC and Monash Institute of Pharmaceutical Sciences, Monash University. JSB is supported by an NHMRC Dementia Leadership Fellowship. MC, JVE, MH and TC are employed by the organisation providing community-based home care services to study participants.

Patient consent for publication Not required.

Ethics approval Monash University Human Research Ethics Committee (approval number 11614) and the Helping Hand ethical review panel.

Provenance and peer review Not commissioned; externally peer reviewed.

Open access This is an open access article distributed in accordance with the Creative Commons Attribution Non Commercial (CC BY-NC 4.0) license, which permits others to distribute, remix, adapt, build upon this work non-commercially, and license their derivative works on different terms, provided the original work is properly cited, appropriate credit is given, any changes made indicated, and the use is non-commercial. See: http:// creativecommons.org/licenses/by-nc/4.0/.

\section{REFERENCES}

1. Morgan TK, Williamson M, Pirotta M, et al. A national census of medicines use: a 24-hour snapshot of Australians aged 50 years and older. Med J Aust 2012;196:50-3.

2. Charlesworth CJ, Smit E, Lee DS, et al. Polypharmacy among adults aged 65 years and older in the United States: 1988-2010. J Gerontol A Biol Sci Med Sci 2015;70:989-95.

3. Sluggett JK, Stasinopoulos J, Bell JS. Medication management by informal caregivers - Under-recognized and under-supported. Res Social Adm Pharm 2018;14:215-7.

4. Maddigan SL, Farris KB, Keating N, et al. Predictors of older adults' capacity for medication management in a self-medication program: a retrospective chart review. J Aging Health 2003;15:332-52.

5. Wimmer BC, Bell JS, Fastbom J, et al. Medication regimen complexity and number of medications as factors associated with unplanned hospitalizations in older people: a population-based cohort study. J Gerontol A Biol Sci Med Sci 2016;71:831-7.

6. Wimmer BC, Bell JS, Fastbom J, et al. Medication regimen complexity and polypharmacy as factors associated with all-cause mortality in older people: a population-based cohort study. Ann Pharmacother 2016;50:89-95.

7. Sluggett JK, Chen EYH, llomäki J, et al. SImplification of Medications Prescribed to Long-tErm care Residents (SIMPLER): study protocol for a cluster randomised controlled trial. Trials 2018;19:37.

8. Poland F, Mapes S, Pinnock H, et al. Perspectives of carers on medication management in dementia: lessons from collaboratively developing a research proposal. BMC Res Notes 2014;7:463.

9. Tan ECK, Sluggett JK, Johnell K, et al. Optimizing Geriatric Pharmacotherapy through Pharmacoepidemiology Network (OPPEN) Group. Research priorities for optimizing geriatric pharmacotherapy: an international consensus. J Am Med Dir Assoc 2018;19:193-9.

10. Giron MS, Wang HX, Bernsten C, et al. The appropriateness of drug use in an older nondemented and demented population. J Am Geriatr Soc 2001;49:277-83.

11. Page A, Etherton-Beer C, Seubert LJ, et al. Medication use to manage comorbidities for people with dementia: a systematic review. Journal of Pharmacy Practice and Research 2018;48:356-67.

12. Elliott RA, Goeman D, Beanland C, et al. Ability of older people with dementia or cognitive impairment to manage medicine regimens: a narrative review. Curr Clin Pharmacol 2015;10:213-21.

13. Noureldin M, Plake KS. Correlates of caregivers' involvement in the management of older adults' medications. Res Social Adm Pharm 2017;13:840-8.

14. Page AT, Clifford RM, Potter K, et al. Exploring the enablers and barriers to implementing the Medication Appropriateness Tool for Comorbid Health conditions during Dementia (MATCH-D) criteria in Australia: a qualitative study. BMJ Open 2017;7:e017906.

15. Gillespie R, Mullan J, Harrison L. Managing medications: the role of informal caregivers of older adults and people living with dementia. A review of the literature. J Clin Nurs 2014;23:3296-308.

16. Wroe AL. Intentional and unintentional nonadherence: a study of decision making. J Behav Med 2002;25:355-72.

17. Sluggett JK, llomäki J, Seaman KL, et al. Medication management policy, practice and research in Australian residential aged care: Current and future directions. Pharmacol Res 2017;116:20-8.

18. Eldridge SM, Lancaster GA, Campbell MJ, et al. Defining feasibility and pilot studies in preparation for randomised controlled trials: development of a conceptual framework. PLoS One 2016;11:e0150205.

19. O'Cathain A, Hoddinott $P$, Lewin S, et al. Maximising the impact of qualitative research in feasibility studies for randomised controlled trials: guidance for researchers. Pilot Feasibility Stud 2015;1:32.

20. Chan AW, Tetzlaff JM, Altman DG, et al. SPIRIT 2013 statement: defining standard protocol items for clinical trials. Ann Intern Med 2013;158:200-7.

21. Commonwealth of Australia. Commonwealth Home Support Programme. https://www.myagedcare.gov.au/help-home/ commonwealth-home-support-programme (accessed 15 June 2018).

22. Australian Government Department of Health. About the Home Care Packages Program. https://agedcare.health.gov.au/programs/homecare/about-the-home-care-packages-program (accessed 15 Jun 2018).

23. Australian Institute of Health and Welfare. Dementia in Australia. Cat. no. AGE 70. Canberra: AlHW 2012.

24. National Health and Medical Research Council, Australian Research Council and Universities Australia. National statement on ethical conduct in human research 2007 (Updated 2018. Canberra: Commonwealth of Australia, 2007. updated 2018.

25. Australian Government Department of Health. Dementia language guidelines. https://www.dementia.org.au/resources/dementialanguage-guidelines (accessed 15 Jun 2018). 
26. Dementia Australia Dementia Advisory Committee. Talk to Me. https://www.dementia.org.au/research-and-publications/papers-andevaluations/talk-to-me (accessed 15 Jun 2018).

27. Pronovost $\mathrm{P}$, Weast $\mathrm{B}$, Schwarz $\mathrm{M}$, et al. Medication reconciliation: a practical tool to reduce the risk of medication errors. J Crit Care 2003;18:201-5.

28. Rose AJ, Fischer SH, Paasche-Orlow MK. Beyond medication reconciliation: the correct medication list. JAMA 2017;317:2057-8.

29. Boyce LE, Knight AT. Audit of general practitioner medication lists for older patients at a regional hospital. Journal of Pharmacy Practice and Research 2013;43:105-8.

30. Chen EY, Sluggett JK, Ilomäki J, et al. Development and validation of the Medication Regimen Simplification Guide for Residential Aged CarE (MRS GRACE). Clin Interv Aging 2018;13:975-86.

31. Edelberg HK, Shallenberger E, Wei JY. Medication management capacity in highly functioning community-living older adults: detection of early deficits. J Am Geriatr Soc 1999;47:592-6.

32. Braun V, Clarke V. Using thematic analysis in psychology. Qual Res Psychol 2006;3:77-101.

33. Risser J, Jacobson TA, Kripalani S. Development and psychometric evaluation of the Self-efficacy for Appropriate Medication Use Scale (SEAMS) in low-literacy patients with chronic disease. J Nurs Meas 2007:15:203-19.

34. Logsdon RG, Gibbons LE, McCurry SM, et al. Quality of life in Alzheimer's disease: patient and caregiver reports. J Ment Health Aging 1999;5:21-32.

35. Hawthorne G, Sansoni J, Hayes L, et al. Measuring patient satisfaction with health care treatment using the Short Assessment of Patient Satisfaction measure delivered superior and robust satisfaction estimates. J Clin Epidemiol 2014;67:527-37.

36. Billingham SA, Whitehead AL, Julious SA. An audit of sample sizes for pilot and feasibility trials being undertaken in the United Kingdom registered in the United Kingdom Clinical Research Network database. BMC Med Res Methodol 2013;13:104.
37. Campbell M, Fitzpatrick R, Haines A, et al. Framework for design and evaluation of complex interventions to improve health. BMJ 2000;321:694-6.

38. Drenth-van Maanen AC, Spee J, van Hensbergen L, et al. Structured history taking of medication use reveals iatrogenic harm due to discrepancies in medication histories in hospital and pharmacy records. J Am Geriatr Soc 2011;59:1976-7.

39. Beer C, Flicker L, Horner B, et al. Factors associated with self and informant ratings of the quality of life of people with dementia living in care facilities: a cross sectional study. PLoS One 2010;5:e15621.

40. Sixth Community Pharmacy Agreement. Home Medicines Review. http://6cpa.com.au/medication-management-programs/homemedicines-review/ (accessed 5 Jun 2019).

41. Morley JE, Malmstrom TK, Miller DK. A simple frailty questionnaire (FRAIL) predicts outcomes in middle aged African Americans. J Nutr Health Aging 2012;16:601-8.

42. Kojima G. Frailty defined by frail scale as a predictor of mortality: a systematic review and meta-analysis. J Am Med Dir Assoc 2018;19:480-3.

43. Clark CM, Ewbank DC. Performance of the dementia severity rating scale: a caregiver questionnaire for rating severity in Alzheimer disease. Alzheimer Dis Assoc Disord 1996;10:31-9.

44. Dementia Centre for Research Collaboration. DementiaKT. http:// dementiakt.com.au/doms/2018 (accessed 15 June 2018).

45. Katz S, Ford AB, Moskowitz RW, et al. Studies of illness in the aged. the index of adl: a standardized measure of biological and psychosocial functiON. JAMA 1963;185:914-9.

46. Logsdon RG, Gibbons LE, McCurry SM, et al. Assessing quality of life in older adults with cognitive impairment. Psychosom Med 2002;64:510-9.

47. Commonwealth of Australia. Australian Commission on Safety and Quality in Health Care. Preventing falls and harm from falls in older people: Best practice guidelines for Australian residential aged care facilities. Canberra: Commonwealth of Australia, 2009. 